\title{
Olbia Pontica. Principaux résultats des fouilles menées de 2006 à 2010
}

\section{Valentina Vladimirovna Krapivina}

Traducteur : Yves Guignard

\section{OpenEdition \\ Journals}

Édition électronique

URL : http://journals.openedition.org/edl/358

DOI : $10.4000 /$ edl.358

ISSN : 2296-5084

\section{Éditeur}

Université de Lausanne

\section{Édition imprimée}

Date de publication : 15 mai 2012

Pagination : 261-278

ISBN : 978-2-940331-27-7

ISSN : 0014-2026

\section{Référence électronique}

Valentina Vladimirovna Krapivina, « Olbia Pontica. Principaux résultats des fouilles menées de 2006 à 2010 », Études de lettres [En ligne], 1-2 | 2012, mis en ligne le 15 mai 2015, consulté le 19 décembre 2020. URL : http://journals.openedition.org/edl/358 ; DOI : https://doi.org/10.4000/edl.358 


\section{OLBIA PONTICA \\ PRINCIPAUX RÉSULTATS DES FOUILLES MENÉES \\ DE 2006 À 2010}

Entre 2006 et 2010, les investigations de terrain menées à Olbia Pontica ont été entreprises dans les secteurs particulièrement endommagés par les éléments naturels. Le secteur R-25 au sud-est de la ville haute, la zone des portes de ville sur l'enceinte septentrionale de la citadelle, le secteur T-3 dans sa partie en terrasses, le secteur NGS au nord de la ville basse et deux secteurs à l'endroit de la nécropole figurent au nombre des principales surfaces fouillées à Olbia.

Olbia Pontica comptait au nombre des trois plus grandes cités du littoral septentrional de la mer Noire. Son intérêt archéologique ne réside pas tant dans le rôle majeur qu'elle a joué pour cette région dans l'Antiquité, que dans l'excellente préservation des couches d'occupation grecque. A l'heure actuelle, les niveaux se rapportant à cette période sont peut-être les plus exhaustivement étudiés de toute la région. La particularité d'Olbia réside dans le fait que le site ne présente aucune occupation médiévale ou moderne, à la différence des autres établissements antiques d'importance fondés sur la côte septentrionale de la mer Noire. La dégradation du gisement archéologique d'Olbia remonte à une période récente. Tout d'abord, la forteresse d'Očakov fut construite par les Turcs en utilisant les matériaux de la ville antique; plus tard, suite à l'annexion de ces territoires par l'Empire russe, le village voisin de Parutino fut créé et ses habitants utilisèrent des pierres d'Olbia pour la construction de leurs maisons ${ }^{1}$.

I. S. D. Kryjitskij, V. V. Krapivina, "A Quarter-Century of Excavations at Olbia Pontica», p. 181 sq. 
Olbia fut fondée par des colons grecs en provenance de Milet sur la rive droite de l'estuaire du Boug méridional (près du village actuel de Parutino, dans la région de Nikolaev [Ukraine]), à la charnière du premier et du deuxième quart du $\mathrm{VI}^{\mathrm{e}} \mathrm{s}$. av. J.-C., et elle a subsisté pendant près de mille ans, jusqu'au dernier quart du $\mathrm{IV}^{\mathrm{e}}$ s. apr. J.-C. Olbia jouissait d'une renommée étendue dans le monde classique et entretenait des liens étroits avec lui. Une de ses particularités réside encore dans le fait que la cité était entourée de tribus barbares, scythes tout d'abord, puis sarmates et enfin des tribus de la culture dite de Černjakhov.

Aujourd'hui, la superficie du Parc national historique et archéologique «Olbia» de l'Académie des sciences d'Ukraine occupe 270 hectares: le territoire préservé de la cité compte 33 hectares et sa nécropole près de 240 hectares. En outre près de 20 hectares de la cité sont recouverts par l'estuaire du Boug méridional.

Le territoire d'Olbia se divise en trois parties: la ville haute, située entre $30 \mathrm{~m}$ et $42 \mathrm{~m}$ en dessus du niveau de l'estuaire, la ville basse située entre $4 \mathrm{~m}$ et $10 \mathrm{~m}$ en dessus de l'estuaire, et la terrasse qui relie la ville haute à la ville basse. A en juger par les ruines du mur d'enceinte fortifié au nord, repéré dans l'estuaire, la cité s'étendait entre $170 \mathrm{~m}$ à $200 \mathrm{~m}$ à l'est de la rive actuelle. Les frontières ouest, nord et sud d'Olbia coïncident globalement avec celles que l'on peut observer actuellement dans le terrain.

Des fouilles systématiques ont été menées à Olbia chaque année depuis plus d'un siècle. L'histoire de ces investigations archéologiques se divise en trois étapes, correspondant aux différents buts que se fixèrent trois générations de chercheurs ${ }^{2}$. Suite aux investigations entreprises depuis lors, quelque six hectares du territoire de la cité antique ont été exhumés, ainsi qu'une petite partie de sa nécropole.

Deux téménos comprenant les ruines de temples et d'autels, une agora ceinte de bâtiments officiels, des quartiers d'habitation et des zones de production artisanale, des fortifications et bien d'autres structures y ont été mis en évidence. Ainsi, Olbia livre chaque année de nouvelles découvertes sur la culture antique - des statues en marbre, des inscriptions, des figures en terre cuite, différents vases, des pièces de monnaie, etc.

Durant plus de trois décennies, les fouilles menées dans une perspective de recherches scientifiques se sont d'abord concentrées sur

2. Ibid., p. 182-184. 
les zones particulièrement endommagées par l'érosion, les glissements de terrain et d'autres facteurs naturels, ainsi que sur les endroits touchés par le pillage avant 2003, date à laquelle un garde permanent fut installé dans le Parc d'Olbia. Le secteur R-25 au sud-est de la ville haute, le secteur du mur fortifié au nord de la citadelle, le secteur T-3 dans sa partie en terrasses, le secteur NGS au nord de la ville basse (fig. 1) et deux secteurs de la nécropole ont ainsi été privilégiés pour la fouille.

\section{Fouilles dans la ville haute}

\section{Secteur R-25}

(sous la direction de V. V. Krapivina et de A. V. Bujskikh)

Ce secteur est situé au sud-ouest de la ville haute d'Olbia, en dessus de l'estuaire, sur le territoire que l'on désigne comme celui de la citadelle romaine (fig. 1.1). C'est maintenant l'un des secteurs les plus fouillés de la cité antique. Des investigations scientifiques, dont les résultats ont été régulièrement publiés ${ }^{3}$, s'y déroulent sans interruption depuis 1982. Cette partie de la ville fut habitée dès la fin du premier quart du $\mathrm{VI}^{\mathrm{e}} \mathrm{s}$. av. J.-C. et jusque dans les années 380 apr. J.-C. Les fouilles menées durant les années 2006 à 2010 ont concerné surtout le sud du secteur et un peu le nord, le long de la berge de l'estuaire. Elles ont révélé deux terrasses, une supérieure et une inférieure.

Au cours du deuxième quart du $\mathrm{VI}^{\mathrm{e}}$ s. av. J.-C., des cabanes enterrées et semi-enterrées furent bâties sur ce territoire; le peuplement d'Olbia peut d'ailleurs bien être parti de ce point-là. Près de trente cabanes enterrées et semi-enterrées y ont été signalées de même qu'un grand nombre de fosses de la période archaïque. Ces premières maisons des Olbiopolitains étaient rondes, ovales et rectangulaires. La plupart d'entre elles servaient à l'habitat, d'autres avaient une fonction domestique. Certaines étaient munies d'éléments de charnière métalliques (pl. XXVI.1). Toutes furent

3. V. V. Krapivina, «Kratkie itogi rabot na učastke R-25 v Ol’vii v 2000-2001 godakh" et "Issledovanija poslednikh let v južnoj časti Verkhnego i Nižnego goroda Ol'vii»; V. V. Krapivina, A. V. Bujskikh, "Predvaritel'nye itogi issledovanija jugovostočnoj časti Verkhnego goroda Ol'vii (1982-1996)", "Issledovanija v jugo-vostočnoj časti rimskoj citadeli Ol’vii (učastok R-25) v 2004-2005 gg. " et "Osnovnye raboty na učastke R-25 v Ol'vii v 2007-2008 gg. ». 
Fig. 1 - Plan topographique d'Olbia:

1 - secteur R-25; 2 - secteur L-1; 3 - secteur du mur nord de la citadelle; 4 - secteur B-III ; 5 - secteur T-3; 6 - secteur NGS. 
remblayées de terre durant le premier quart du $\mathrm{V}^{\mathrm{e}}$ s. av. J.-C. Ces dernières années, la quantité de céramiques archaïques à figures noires collectées là a considérablement augmenté.

Il faut également faire état d'une structure dont la fonction d'origine nous est inconnue et qui est datée de la première moitié du $\mathrm{V}^{\mathrm{e}} \mathrm{s}$. av. J.-C. Il s'agit d'une fosse de forme ronde aménagée dans le niveau naturel et qui a les dimensions maximales suivantes: $4,7 \mathrm{mx} 4,5 \mathrm{~m}$ pour $3,6 \mathrm{~m}$ à $4 \mathrm{~m}$ de profondeur. Il est impossible d'y voir un habitat du fait de l'importance de ses dimensions, de sa profondeur inhabituelle et de son absence de constructions additionnelles. Il fut employé par la suite pour y jeter des ordures et, en partie, peut-être comme bothros du sanctuaire d'Aphrodite. Dans un de ses recoins sont apparus des fragments peints de terre cuite architecturale et treize fragments de récipients portant des inscriptions, pour la plupart dédiées à Aphrodite ${ }^{4}$. Par le passé, la partie sud du secteur a révélé un bothros riche d'ex-voto à Aphrodite datables des années 480-410 av. J.-C., ainsi que d'autres objets manufacturés qui attestent l'existence d'un sanctuaire d'Aphrodite au moins depuis la fin du VI ${ }^{\mathrm{e}}$ siècle et jusqu'au $\mathrm{II}^{\mathrm{e}}$ s. av. J.-C.

Au sud du secteur R-25, de nombreuses fosses d'une certaine largeur ont été mises au jour, certaines datant des $\mathrm{VI}^{\mathrm{e}}-\mathrm{V}^{\mathrm{e}}$ s. av. J.-C., d'autres du $\mathrm{I}^{\mathrm{er}}$ au IV $\mathrm{V}^{\mathrm{e}}$ siècle de notre ère (pl. XXVI.2 et XXVII). A l'origine, elles servaient principalement à la conservation du grain. Cette hypothèse a d'ailleurs été confirmée ensuite par des analyses paléobotaniques ${ }^{5}$ - la présence de cavités dues à des rongeurs trahissait déjà une telle fonction. Plus tard, ces structures en creux furent utilisées comme décharges à ordure.

La conservation de la couche archéologique des $\mathrm{I}^{\mathrm{er}}$ et $\mathrm{II}^{\mathrm{e}}$ s. apr. J.-C. est meilleure à cet endroit que dans le reste du secteur. Les complexes rapportés au $\mathrm{I}^{\text {er }}$ siècle et à la première moitié du $\mathrm{II}^{\mathrm{e}}$ s. apr. J.-C. sont rares à Olbia, d'où l'importance particulière qu' ils revêtent à nos yeux.

La partie sud de la ville haute d'Olbia fut nivelée et reconstruite selon un nouveau plan dans la seconde moitié du $\mathrm{II}^{\mathrm{e}}$ s. apr. J.-C., après l'arrivée d'une garnison romaine et tandis que la citadelle était construite à cet endroit. Des terrasses et des murs de soutènement furent bâtis le

4. V. V. Krapivina, A. V. Bujskikh, «Osnovnye raboty na učastke R-25 v Ol'vii v 2007-2008 gg.", p. 114 sq.

5. Elles ont été effectuées par le $\mathrm{D}^{\mathrm{r}} \mathrm{G}$. A. Paškevič. 
long de la pente. C'est ainsi que les couches archéologiques des périodes précédentes furent en grande partie détruites.

Les fouilles entreprises au sud du secteur R-25 ont révélé les ruines d'une maison, bâtie avant la seconde moitié du I ${ }^{\text {er }}$ siècle et ayant perduré jusqu'au III' s. apr. J.-C. (pl. XXVIII.1). Les ruines de cette maison consistent en une chambre à même le sol, deux caves avec des murs conservés sur 2,27 m d'élévation au maximum, ainsi qu'une cour. Sous le pavement de la cour fut creusée une fosse qui a révélé, dans sa partie supérieure, la sépulture d'un enfant ${ }^{6}$. Au nord et à l'est de la maison, quelque vingt fosses de cette période ont été découvertes. Elles étaient remplies d'une grande quantité d'objets, parmi lesquels des vases en céramique intacts, des fragments de verre, des objets métalliques.

Plus de vingt autres fosses ont été datées entre le III et le $\mathrm{IV}^{\mathrm{e}} \mathrm{s}$. apr. J.-C. Aucune n'a révélé un contenu aussi riche que celles datant du I ${ }^{\text {er }}$ et du II ${ }^{\mathrm{e}}$ s. apr. J.-C. Elles contenaient différentes sortes de pierres, des fragments de détails architecturaux, parfois des fragments de statues en marbre. Parmi celles-ci, notons la présence d'un buste en marbre de Mithra, d'une large tête en marbre représentant un homme, peut-être Alexandre le Grand, et d'une tête en marbre d'une statue d'Aphrodite.

Les fouilles ont encore révélé la cave d'une construction complexe taillée dans le niveau naturel à la fin du III e siècle ou au début du $\mathrm{IV}^{\mathrm{e}} \mathrm{s}$. apr. J.-C. Il s'agit d'une excavation ovale de $2,53 \mathrm{~m} \times 2,38 \mathrm{~m}$ et d'une profondeur conservée de $65 \mathrm{~cm}$ à $140 \mathrm{~cm}$. Dans sa partie occidentale, on a mis au jour une fosse conique de $153 \mathrm{~cm}$ x $150 \mathrm{~cm}$ et d'une profondeur de $2,15 \mathrm{~m}$.

Sur la terrasse inférieure de la partie sud du secteur, les fouilles ont révélé un mur de soutènement pour la terrasse supérieure, datant du I I $\mathrm{II}^{\mathrm{e}}$ s. apr. J.-C. qui avait été construit pour intégrer le système de murs de soutènement excavés là au préalable. Quelques fosses étaient situées plus à l'ouest sur la terrasse supérieure. On les a remplies par la suite au moyen de terre et de cailloux et des fortifications furent érigées à cet endroit à la fin du $\mathrm{II}^{\mathrm{e}}$ siècle. Le mur de soutènement existait toujours à cette époque-là et il fut utilisé pour construire la façade extérieure du mur d'enceinte fortifié (pl. XXVIII.2). A l'extérieur se trouvaient des lieux de déversement des ordures. Les couches archéologiques à cet endroit sont

6. V. V. Krapivina, A. V. Bujskikh, «Osnovnye raboty na učastke R-25 v Ol’vii v 2007-2008 gg. ", p. 110-113. 
en effet riches en mobilier. Au IV s. apr. J.-C., un atelier métallurgique prit place par-dessus. Une seule pierre de mur et une fosse remplie de déchets d'une production de plomb et de bronze ont été préservées.

La plupart des objets trouvés ces dernières années dans la partie nord du secteur R-25 datent de la fin du III $-I^{\mathrm{e}}$ s. apr. J.-C. C'est le premier endroit à Olbia où une couche archéologique de cette période est mise au jour sur une si grande surface. Elle recelait les vestiges de nombreuses maisons, de rues et même de places pavées.

Trois phases de construction peuvent être retracées ici. Les caractéristiques de la construction diffèrent un peu des époques précédentes. Comme auparavant, la pente a été terrassée et les maisons construites respectivement sur les terrasses inférieure et supérieure. Les maisons étaient plutôt grandes, de forme rectangulaire, hautes parfois de deux étages à en juger par l'épaisseur des murs. Les locaux destinés à l'habitation étaient combinés avec les locaux à usage domestique et avec les ateliers ${ }^{7}$. Manifestement, Olbia ne possède pas de fortifications correspondant à la dernière période de son existence. Elles furent en partie détruites durant la mise à sac de la ville par les Goths en 269-270 et n'ont pas été reconstruites par la suite. Des blocs de pierre du mur de fortification situé à l'est ont été découverts dans des habitations du IVe $s$. apr. J.-C. Ces dernières années, des canalisations du IVe s. apr. J.-C. ont été révélées à l'endroit de fortifications, le long de la pente en dessus de l'estuaire.

En 2007, au nord-ouest du secteur R-25, une partie de l'allée principale traversant Olbia dans sa longueur fut mise au jour; ses dimensions sont de $21 \mathrm{~m}$ sur $2 \mathrm{~m}$ à $6 \mathrm{~m}$ (pl. XXIX.1). Elle est orientée, comme dans les époques plus anciennes, du nord au sud. Le pavement est constitué de dalles de pierre, de pierres brisées, de fragments de céramique et d'ossements d'animaux liés par un mortier d'argile, mélangé à du sable et de la chaux. L'emploi d'ossements d'animaux et de fragments de céramique pourrait témoigner qu’on est allé puiser dans la décharge publique pour cette construction. L'allée s'élargit au sud pour former une place. C'est à cet endroit qu'a été découvert un statère en bronze de Rheskouporis V (325-326).

7. V. V. Krapivina, «O zastrojke Ol’vii v kontse III-IV vv. n.è.». 
Secteur L-1

(sous la direction de V. V. Krapivina et de D. N. Khmelevskij)

On a procédé entre 2003 et 2006, dans le secteur L-1 (fig. 1.2), à des fouilles préventives allant jusqu'au bâtiment du praetorium ${ }^{8}$. Sa surface est aujourd'hui de près de $140 \mathrm{~m}^{2}$. A une profondeur de $20 \mathrm{~cm}$ à $45 \mathrm{~cm}$ sous le niveau actuel, une large route du IVe s. apr. J.-C. a été mise au jour (pl. XXIX.2). Elle coupe perpendiculairement la route principale; sa largeur oscille entre $7,8 \mathrm{~m}$ et $8,8 \mathrm{~m}$, et sa longueur mise au jour atteint les $15 \mathrm{~m}$. Sous la partie nord de la route, les fouilles ont révélé les vestiges du pavement de la cour du bâtiment ainsi que les ruines d'un atelier viticole de la fin du II ${ }^{e}$-III e s. apr. J.-C. Au sud du secteur, elles ont révélé un pavement de céramique en opus spicatum, réalisé d'après la technique de construction des Romains, mais à partir de fragments de tuiles (pl. XXX.1). C'est le premier opus de ce genre découvert à Olbia.

Secteur du mur nord de la citadelle

(sous la direction de S. D. Kryžitskij et de D. N. Khmelevskij)

On procède à des fouilles à cet endroit depuis 2005 dans le but de reconstituer la porte nord de la citadelle (fig. 1.3). Les vestiges des portes de la citadelle et des tours qui la flanquaient n'avaient pas été découverts à l'issue des fouilles de B. V. Farmakovskij en 1904, 1905 et 1924. Leur emplacement a été déterminé par l'allée principale traversant la ville dans sa longueur et qui descend de la citadelle depuis le nord. Les fouilles de ces dernières années confirment l'absence des tours sur les deux côtés de l'allée. En outre, les recherches entreprises ont mis au jour une construction qui consiste en un pilier massif de forme rectangulaire $(1,95 \mathrm{~m}$ x $2 \mathrm{~m}$ ) ceint d'un pavement à trois couches sur ses côtés sud et ouest affectant la forme d'un quart de cercle aménagé dans le niveau naturel sur plus d'un mètre. Rien n'en a été mentionné dans les rapports de B. V. Farmakovskij et ces éléments n'ont manifestement pas été découverts par lui (pl. XXX.2). Une telle construction servait probablement de

8. V. V. Krapivina, D. N. Khmelevskij, "Okhrannye raboty na učastkakh L-1 i NGF v Ol'vii v 2004-2005 godakh». 
fondation aux escaliers de la "Tour-en-dessus-de-la-porte» ${ }^{9}$. Les fouilles ont révélé ici des couches datant principalement des $\mathrm{II}^{\mathrm{e}}$ et $\mathrm{III}^{\mathrm{e}} \mathrm{s}$. apr. J.-C. Enfin, pendant les fouilles, il s'est avéré que le bâtiment décrit par B. V. Farmakovskij comme une caserne - et dont il estimait qu'il était connecté au système de fortification - a en réalité été construit à une époque antérieure (pl. XXXI.1).

Entre 2009 et 2010, les fouilles se sont poursuivies sur une surface de $120 \mathrm{~m}^{2}$ à l'ouest du pilier et de la route principale qui traverse la ville dans sa longueur. L'objectif des investigations était de mettre au jour la continuation du mur nord de la citadelle. On y a découvert des amas de pierres et des fosses. La plupart des objets ont pu être datés du Irr siècle $\mathrm{au} \mathrm{IV}^{\mathrm{e}}$ s. apr. J.-C., bien qu'un certain nombre de découvertes soient plus anciennes, à savoir du $\mathrm{VI}^{\mathrm{e}}$ siècle au $\mathrm{I}^{\mathrm{er}} \mathrm{s}$. av. J.-C.

Secteur B-III

(sous la direction de S. D. Kryžitskij et de V. I. Nazarčuk)

Ces fouilles préventives ont été entreprises en 2009 dans la partie nord d'Olbia (fig. 1.4) sur une surface de $40 \mathrm{~m}^{2}$. Elles ont révélé l'angle sudouest d'une maison et une partie de la rue dont le pavement date de la période hellénistique. Sous la maison, on a dégagé une couche de loess atteignant jusqu'à $2 \mathrm{~m}$ d'épaisseur pour trouver, en dessous, une couche de déchets datant de la seconde moitié $\mathrm{du} \mathrm{V}^{\mathrm{e}}$ siècle à la première moitié du IVe s. av. J.-C.

\section{Zone en terrasse}

Secteur T-3

(sous la direction de S. D. Kryžitskij et de V. I. Nazarčuk)

Les fouilles furent menées de 1989 à 2003 à l'est du téménos (fig. 1.5) dans l'espoir de dégager le théâtre d'Olbia, mentionné sur des inscriptions locales. Les vestiges d'une structure d'une seule pièce équipée d'un

9. S. D. Kryžitskij, D. N. Khmelevskij, «K voprosy o komplekse krepostnykh vorot rimskoj cidadeli v Ol'vii » et "Okhrannye dosledovanija severnoj oboronitel'noj linii rimskoj citadeli Ol'vii (2006-2007 gg.)", p. 138 sq. et 141. 
foyer, datant de la fin du $\mathrm{VI}^{\mathrm{e}}$ siècle au début du $\mathrm{V}^{\mathrm{e}}$ s. av. J.-C., y apparurent. Il s'agit du plus ancien bâtiment en surface repéré dans la zone en terrasse de la ville. On a découvert encore dans ce secteur une grande maison bien conservée de la période hellénistique, avec sa cour pavée de dalles de pierre. C'est ici que des recherches ont été effectuées sur tout un complexe de tuiles couvrantes, certaines estampillées et d'autres peintes. L'occupation la plus récente de l'endroit remonte aux trois premiers siècles apr. J.-C. environ ${ }^{10}$.

En 2009-2010, sur une surface de $190 \mathrm{~m}^{2}$, des fouilles ont été conduites sur une surface s'étendant à l'est du secteur. Elles avaient pour but de mettre au jour l'intégralité de la maison hellénistique et les limites de la terrasse. On y a découvert d'importants amas de pierres et des fragments de maçonnerie en moellon. Manifestement, la maçonnerie en question est liée aux ruines d'un faubourg datant du $\mathrm{IV}^{\mathrm{e}}$ s. apr. J.-C. (pl. XXXI.2).

Des travaux mineurs ont été effectués au nord-est des fondations de la maison hellénistique sur une surface de $10 \mathrm{~m}^{2}$; la partie orientale de ces fondations a été endommagée par un éboulement ancien.

\section{Fouilles dans la ville basse}

Le secteur NGS nord (sous la direction de N. A. Lejpunskaja, T. L. Samojlova, puis plus tard de A. V. Karjaka et P. D. Diatroptov) se situe tout au nord-est de la ville basse d'Olbia (fig. 1.6 et pl. XXXII). Le résultat des fouilles effectuées entre 1985 et 2004 a été récemment publié par un collectif sous la forme d'une monographie ${ }^{11}$. Les fouilles s'y sont poursuivies de 2005 à 2008. Elles se sont concentrées sur les parties nord et sud du secteur.

$\mathrm{Au}$ nord, on a découvert les vestiges du mur de fortification septentrional, lui-même orienté d'ouest en est. Outre la tranchée de fondation, on note parmi les restes de cette construction, une structure

Io. S. D. Kryžitskij, V. I. Nazarčuk, « Novyj pamjatnik stroitel'stva pozdnearkhaičeskoj Ol'vii »; V. I. Nazarčuk, "Osnovnye rezul’taty raskopok učastka T-3 v Ol'vii (1989-1995)».

II. N. A. Lejpunskaja, P. Guldager Bilde, J. Munk Højte, V. V. Krapivina, S. D. Kryzickij (eds), The Lower City of Olbia (sector NGS) in the 6th Century BC to the 4th Century AD. 
de fondation en argile jaune, une partie de la maçonnerie du mur et des amas de gravats. Le mur de fortification a été construit au début du $\mathrm{III}^{\mathrm{e}}$ s. av. J.-C. sur la couche archéologique du $\mathrm{IV}^{\mathrm{e}}$ s. av. J.-C. C'est à cet emplacement que les archéologues ont dégagé une couche incendiée, des résidus d'ossements humains et de crânes, manifestement en lien avec le siège de Zopyrion en 331-330 av. J.-C. ${ }^{12}$.

Au sud, on a mis au jour des maisons et des pavements de l'époque hellénistique, que les fouilles précédentes avaient en partie mis à nu. La longueur totale de la surface pavée est de plus de vingt mètres. Aucune ruine de bâtiment n'a été mise au jour au sud-est. Il s'agissait probablement de la limite des terrassements avec une importante différence de niveau par rapport à l'estuaire.

\section{Fouilles de la nécropole}

Des fouilles ont été menées depuis de nombreuses années dans les parties est et nord de la nécropole, aux caps 1 et 2 (sous la direction de Ju. I. Kozub) ${ }^{13}$. Dans la partie nord de la nécropole, on a découvert le faubourg de la période classique. Les limites de cette banlieue ont pu être définies. Par la suite, le territoire de la nécropole s'est étendu jusqu'ici. On a découvert dans la partie nord-est de la nécropole un site sacré avec différents types d'autels, façonnés dans la terre ou en pierre, ainsi que des coupes spécifiques aux libations.

De 2006 à 2010, les fouilles se sont poursuivies sur les caps 1 et 2 (sous la direction de A. V. Ivčenko). Sur le cap 1 furent découvertes des cabanes enterrées et semi-enterrées et plus de 20 fosses des $\mathrm{V}^{\mathrm{e}}$ s. et $\mathrm{IV}^{\mathrm{e}} \mathrm{s}$. av. J.-C. ${ }^{14}$. Trois en particulier, datant de la seconde moitié du IVe $s$. av. J.-C., présentent un intérêt certain puisqu'elles recèlent des squelettes humains dans différentes positions. Par le passé, une telle fosse avait déjà été découverte sur le cap 1 . Il est possible que ces dépouilles, jetées dans des fosses, présentent un lien avec les événements qui secouèrent la cité

I2. N. A. Lejpunskaja, «K voprosu ob oboronitel'nykh sooruženijakh v Nižnem gorode Ol'vii (predvaritel'nye itogi rabot)»; A. V. Karjaka, "The Defense Wall in the Northern Part of the Lower City of Olbia Pontike».

I3. J. I. Kozub, «Istoričeskaja topografija nekropolja Ol'vii».

I4. V. V. Krapivina, A. V. Ivčenko, "Novye nakhodki na učastke Severnyj mys 1 nekropolja Ol'vii». 
au moment du siège de Zopyrion. Un réservoir d'eau a par ailleurs été daté de la première moitié du $\mathrm{IV}^{\mathrm{e}} \mathrm{s}$. av. J.-C., tandis que les ruines d'un bâtiment au parement de pierre sont apparues à la fouille (pl. XXXIII.1). Le bâtiment en question a existé à la charnière des $\mathrm{V}^{\mathrm{e}}$ et $\mathrm{IV}^{\mathrm{e}}$ siècles et jusqu'au milieu du $\mathrm{IV}^{\mathrm{e}}$ s. av. J.-C.; il a en outre été reconstruit à deux reprises.

Sur les caps 1 et 2 et dans la partie nord de la nécropole ${ }^{15}$, cinquante tombes de différents types furent examinées. La plupart d'entre elles remontent aux périodes classique et hellénistique. Bien qu'elles aient généralement été pillées, on y a trouvé des vases en argile rouge et gris à vernis noir, des objets en métal et des verreries, des pièces de monnaie dans la majorité des tombes. Dans un hypogée ont été découverts deux squelettes, l'un d'eux en mauvais état de conservation et l'autre identifié comme celui d'un homme. Le caveau était pillé, mais on a retrouvé plusieurs objets laissés sur place, dont un crochet de carquois d'un style appelé scytho-sarmate datant du $\mathrm{II}^{\mathrm{e}}$ s. av. J.-C. ${ }^{16}$.

On trouve encore plusieurs inhumations pillées dans des caveaux datant du $\mathrm{I}^{\mathrm{er}}$ s. et du $\mathrm{II}^{\mathrm{e}}$ s. apr. J.-C. Dans un de ceux-ci (pl. XXXIV), trois squelettes dont les ossements étaient éparpillés et mélangés (il s'agissait d'un enfant, d'un adolescent et d'un adulte ayant atteint un âge avancé) au milieu de nombreux objets: des perles (plus de 650), des vases en verre, la pipette en verre d'un parfumeur avec un oiseau à son extrémité, des objets en bronze et en fer, des pièces de monnaie.

En 2010, deux caveaux funéraires ont été ouverts au nord de la nécropole. L'un d'eux peut être daté de la fin du $\mathrm{IV}^{\mathrm{e}} \mathrm{s}$. ou de la première moitié du III s. av. J.-C. Bien qu'il ait été pillé, différents objets y ont été découverts tels que des vases en céramique, des objets en bronze, des perles (dont une en or), des anneaux en or et deux boucles d'oreille en or en forme de tête de lion. L'autre caveau a été daté du II ${ }^{\mathrm{e}}$ s. apr. J.-C.

Pendant les années 2003 à 2009, les fouilles ont également concerné la partie nord-ouest de la nécropole (sous la direction de V. A. Papanova et S. N. Ljaško). Elles ont révélé les ruines d'une ferme du IVe s. av. J.-C. couvrant une surface de $1200 \mathrm{~m}^{2}$. L'exploitation servait à l'élevage des

I5. Des fouilles d'urgence furent menées pour fixer les caveaux funéraires qui s'étaient affaissés durant l'hiver.

I6. A. V. Ivčenko, E. G. Karnaukh, «Pogrebenie II v. do n.è. s kolčannym krjučkom iz Ol'vijskogo nekropolja». 
bovins et à la culture des céréales. Auparavant, deux grandes cabanes semi-enterrées servant d'ateliers et plusieurs fosses de grandes dimensions occupaient l'endroit (pl. XXXIII.2). Toutes ces structures ont précédé l'extension de la nécropole jusqu'ici. Ainsi, le territoire de ce que l'on appelle «la nécropole» était le domaine rural le plus proche d'Olbia Pontica, pour le moins, à la fin de la période archaïque et jusqu'au début de la période hellénistique ${ }^{17}$. De nombreuses pièces de monnaie en bronze ont été trouvées durant les fouilles, parmi lesquelles celles frappées à Olbia prévalent.

En 2010, des fouilles ont été reprises dans la partie sud-ouest de la nécropole, non loin du secteur de 1996 (sous la direction de V. A. Papanova et S. N. Ljaško) et sur une surface de $275 \mathrm{~m}^{2}$. Elles ont permis la mise au jour de vestiges archéologiques en deux endroits. Creusés dans le sol à une profondeur de $35 \mathrm{~cm}$ à $60 \mathrm{~cm}$, des éléments maçonnés, deux caves et des fosses datent des $\mathrm{V}^{\mathrm{e}}$ s. et $\mathrm{IV}^{\mathrm{e}}$ s. av. J.-C. Pendant les fouilles, de nombreuses pièces de monnaie en bronze furent également trouvées, dont la plupart provenait d'Olbia. Il s'agissait probablement aussi du terrain de la ferme située en ces lieux avant l'extension de la nécropole.

C'est sur ces éléments que s'achève le résumé des principales découvertes réalisées à Olbia Pontica et dans sa nécropole entre 2006 et 2010 .

Valentina Vladimirovna Krapivina Institut d'Archéologie d'Ukraine, Kiev

Traduction de l'anglais d'Yves Guignard

I7. S. N. Ljaško, V. A. Papanova, «Ol’vijskaja usad’ba», «Poluzemljanka redkoj konstrukcii s ol'vijskoj usad'by" et "Ol'vijskaja usad'ba IV v. do n.è.». 


\section{BIBLIOGRAPHIE}

IvČenko, Andrej Valerievič, Karnaukh, Evgenij Grigor'evič, «Pogrebenie II v. do n.è. s kolčannym krjučkom iz Ol'vijskogo nekropolja» " «Une tombe de la nécropole d'Olbia du II ${ }^{\mathrm{e}}$ siècle av. notre ère avec un crochet de carquois»], in $\Sigma \Upsilon \mathrm{MBO} \Lambda \mathrm{A}$. Antičnyj mir Severnogo Pričernomor'ja. Novejšie nakhodki i otkrytija $[\Sigma \mathrm{M} M B \mathrm{OAA}$. Le monde antique du littoral septentrional de la mer Noire. Découvertes récentes], otv. red. Aleksandr Aleksandrovič Maslennikov, Nadežda Avksent'evna Gavriljuk, Moskva/Kiev, ID Triumf Print, 2010, vol. 1, p. 82-87.

Karjaka, Aleksandr Vitalievič, "The Defense Wall in the Northern Part of the Lower City of Olbia Pontike", in Meetings of Cultures in the Black Sea Region. Between Conflict and Coexistence, ed. by Pia Guldager Bilde, Jane Hjarl Petersen, Aarhus, Aarhus University Press, 2008, p. 163-180 (Black Sea Studies 8).

Kozub, Julija Ivanovna, «Istoričeskaja topografija nekropolja Ol'vii» ["Topographie historique de la nécropole d'Olbia»], in Antičnaja kul'tura Severnogo Pričernomor' ja [La civilisation antique du littoral septentrional de la mer Noire], otv. red. Sergej Dmitrievič Kryžitskij et al., Kiev, Naukova Dumka, 1988, p. 156-174.

Krapivina, Valentina Vladimirovna, "Kratkie itogi rabot na učastke R-25 v Ol'vii v 2000-2001 godakh» ["Bref bilan des travaux entamés sur le secteur R-25 à Olbia durant les années 2000 et 2001 », in Arkheologični vidkryttja v Ukraïni u 2000-2001 rr. [Découvertes archéologiques en Ukraine en 2000 et 2001], otv. red. Nadežda Avksent'evna Gavriljuk, Kyïv, Instytut arkheologiï NAN Ukraïny, 2002, p. 140-151.

—, «O zastrojke Ol'vii v kontse III-IV vv. n.è» ["A propos d'une construction d'Olbia à la fin du III ${ }^{\mathrm{e}}$ siècle - IV ${ }^{\mathrm{e}}$ siècle av. notre ère»], in Bospor Kimmerijskij i varvarskij mir v period antičnosti i srednevekov'ja. Oıкos [Le Bosphore Cimmérien et le monde barbare durant l'Antiquité et le Moyen Age. L'Oıкos], otv. red. 
Viktor Nikolaevič Zin’ko, Kertch, 2006, p. 180-188 (Bosporskie čtenija 7).

—, "Novye dannye o kul'te Afrodity v Ol'vii» ["Nouvelles données sur le culte d'Aphrodite à Olbia»], in Severnoje Pričernomor'e v èpokhu antičnosti i srednevekov' ja [Le littoral septentrional de la mer Noire durant l'Antiquité et le Moyen Age], otv. red. Nina Petrovna Sorokina, Denis Valer'evič Žuravljov, Moskva, Gosudarstvennij Istoričeskij Muzej, 2006, p. 188-206 (Trudy Gosudarstvennogo Istoričeskogo Muzeja 159).

—, «Issledovanija poslednikh let v južnoj časti Verkhnego i Nižnego goroda Ol'vii » [Recherches menées ces dernières années sur la partie méridionale des villes haute et basse d'Olbia»], in Pontika 2006. Novejšie issledovanija grečeskikh kolonij Severnogo Pričernomor'ja [Pontika 2006. Recherches récentes sur les colonies grecques du littoral septentrional de la mer Noire], ed. by Ewdoksia Papuci-Władyka, Kraków, Jagellonian University, 2008, p. 95-110.

Krapivina, Valentina Vladimirovna, Bujsкikн, Alla Valerievna, "Predvaritel'nye itogi issledovanija jugo-vostočnoj časti Verkhnego goroda Ol’vii (1982-1996)» [Résultats intermédiaires des recherches menées sur la partie sud-est de la ville haute d'Olbia (1982-1996)»], in Nikonij i antičnyj mir Severnogo Pričernomor'ja [Nikonion et le monde antique du littoral septentrional de la mer Noire], otv. red. Sergej Borisovič Okhotnikov, Odessa, Izdatel'stvo Odesskogo arkheologičeskogo Muzeja, 1997, p. 122-127.

—, «Issledovanija v jugo-vostočnoj časti rimskoj citadeli Ol'vii (učastok R-25) v 2004-2005 gg.» " Recherches menées dans la partie sud-est de la citadelle romaine d'Olbia (Secteur R-25) en 20042005 », in Arkheologični doslidžennja v Ukraïni 2004-2005 rr. [Recherches archéologiques en Ukraine durant les années 2004 et 2005], otv. red. Nadežda Avksent'evna Gavriljuk, Kyïv/Zaporižja, Instytut arkheologiï NAN Ukraïny, 2006, p. 230-237.

—, "Osnovnye raboty na učastke R-25 v Ol’vii v 2007-2008 gg. "[«Principaux travaux menés dans le secteur R-25 d'Olbia

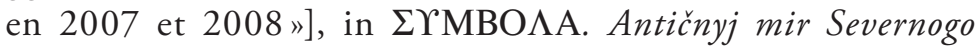

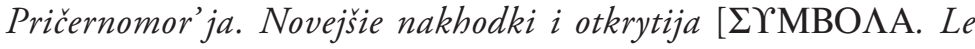
monde antique du littoral septentrional de la mer Noire. Découvertes 
récentes»], otv. red. Aleksandr Aleksandrovič Maslennikov, Nadežda Avksent'evna Gavriljuk, Moskva/Kiev, ID Triumf Print, 2010, vol. 1, p. 108-117.

Krapivina, Valentina Vladimirovna, Ivčenko, Andrej Valerievič, "Novye nakhodki na učastke Severnyj mys 1 nekropolja Ol'vii » ["Nouvelles découvertes effectuées dans le secteur "cap nord 1 " de la nécropole d'Olbia»], in Arkheologični doslidžennja $v$ Ukraïni $2009 r$. [Recherches archéologiques en Ukraine durant l'année 2009], Kyïv/Luc'k, DP «Volyns'ki starožytnosti», 2010, p. $235-237$.

Krapivina, Valentina Vladimirovna, Khmelevskij Dmitrij Nikolaevič, "Okhrannye raboty na učastkakh L-1 i NGF v Ol'vii v 20042005 godakh» ["Travaux de fouille programmés dans les secteurs L-1 et NGF d'Olbia durant les années 2004 et 2005»], in Arkheologični doslidžennja v Ukraïni 2004-2005 rr. [Recherches archéologiques en Ukraine durant les années 2004 et 2005], otv. red. Nadežda Avksent'evna Gavriljuk, Kyïv/Zaporižžja, Instytut arkheologiï NAN Ukraïny, 2006, p. 238-241.

Kryjitskij, Sergej Dmitrievič, Krapivina, Valentina Vladimirovna, "A Quarter-Century of Excavations at Olbia Pontica», Classical Views. University of Calgary Press, 38/2 (1994), p. 181-205.

Kryžitskij, Sergej Dmitrievič, Khmelevskij, Dmitrij Nikolaevič, «K voprosu o komplekse krepostnykh vorot rimskoj cidadeli v Ol'vii » ["Sur la question du complexe archéologique des portes fortifiées de la citadelle romaine d'Olbia»], in Bospor Kimmerijskij i varvarskij mir v period antičnosti i srednevekov' ja. Militaria [Le Bosphore Cimmérien et le monde barbare durant l'Antiquité et le Moyen Age. Militaria], otv. red. Viktor Nikolaevič Zin'ko, Kertch, 2008, p. 147-154 (Bosporskie čtenija 9).

—, "Okhrannye dosledovanija severnoj oboronitel'noj linii rimskoj citadeli Ol'vii (2006-2007 gg.)» ["Recherches programmées de la ligne fortifiée nord de la citadelle romaine d'Olbia (durant les

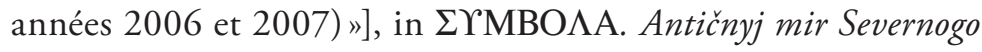

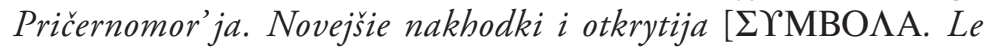
monde antique du littoral septentrional de la mer Noire. Découvertes récentes], otv. red. Aleksandr Aleksandrovič Maslennikov, Nadežda Avksent'evna Gavriljuk, Moskva/Kiev, ID Triumf Print, 2010, vol. 1, p. 137-141. 
Kryžıtskij, Sergej Dmitrievič, Nazarčuk, Vladimir Ivanovič, «Novyj pamjatnik stroitel'stva pozdnearkhaičeskoj Ol'vii» ["Nouveau monument de construction de l'Olbia archaïque tardive»], in Drevnee Pričernomor'e. Kratkie soobščenija Odesskogo arkheologičeskogo ob̌̌čestva [Le littoral pontique ancien. Brèves communications de la société archéologique d'Odessa], otv. red. Sergej Borisovič Okhotnikov, Odessa, Izdatel'stvo Odesskogo arkheologičeskogo Muzeja, 1994, p. 99-106.

Lejpunskaja, Nina Aleksandrovna, "K voprosu ob oboronitel'nykh sooruženijakh v Nižnem gorode Ol'vii (predvaritel'nye itogi rabot)" ["A propos des fortifications de la ville basse d'Olbia (bilan intermédiaire des travaux) »], Bosporskie issledovanija, 17 (2007), p. 48-57.

Lejpunskaja, Nina Aleksandrovna, Guldager Bilde, Pia, Munk Højte, Jakob, Krapivina, Valentina Vladimirovna, KrYzickij, Sergej Dmitrievič (eds), The Lower City of Olbia (sector NGS) in the 6th Century BC to the 4th Century AD, 2 vols, Aarhus, Aarhus University Press, 2010 (Black Sea Studies 13).

LjašKo, Svetlana Nikolaevna, Papanova, Valentina Anatol'evna, «Ol'vijskaja usad'ba (itogi raskopok 2003-2005 gg.) " [ Une villa à Olbia (bilans des fouilles des années 2003 à 2005) »], in Drevnee Pričernomor'e. Sbornik statej, posvjaščennykh 85-letiju professora P. O. Karyškovskogo [Le littoral pontique ancien. Recueil d'articles publié à l'occasion du $85^{e}$ anniversaire du professeur P. O. Karyškovskij], otv. red. Vjačeslav Grigor'evič Kušnir, Odessa, Germes, 2006, p. 134-140.

—, «Poluzemljanka redkoj konstrukcii s ol'vijskoj usad’by» ["Une cabane semi-enterrée de construction rare issue de la villa d'Olbia»], in Drevnee Pričernomor'e [Le littoral pontique ancien], otv. red. Irina Viktorovna Nemčenko, Odessa, Fridman A. S., 2008, vol. 3, p. 223-229.

—, «Ol'vijskaja usad'ba IV v. do n.è.» [«La villa d'Olbia du IV e s. de notre ère»], Starožitnosti stepovogo Pryčornomor' ja i Krymu [Les antiquités du littoral pontique steppique et de la Crimée], otv. red. Pjotr Petrovič Toločko, Zaporižžja, Zaporižskij Natsional'nij Universitet, 2009, vol. 15, p. 100-106.

NAZarČUk, Vladimir Ivanovič, "Osnovnye rezul'taty raskopok učastka T-3 v Ol'vii (1989-1995)» ["Principaux résultats des fouilles 
du secteur T-3 d'Olbia (1989-1995)»], in Istorija. Etnografija. Kul'tura. Novi doslidžennja. Tezy peršoi oblasnoi naukovoi kraeznavcoi konferencii [Histoire, Ethnographie, Culture. Recherches récentes. Préactes du premier colloque scientifique d'études régionales], Mykolaïv, Možlivosti Kimmeriï, 1995, p. 36-38.

Crédits iconographiques

Fig. 1:

Plan de D. B. Belen'kij.

Pl. XXVI-XXXI :

Photos de V. V. Krapivina.

Pl. XXXII:

Photo de S. D. Kryžitskij.

Pl. XXXIII.1 et XXXIV :

Photos de A. V. Ivčenko.

Pl. XXXIII.2:

Photo de V. A. Papanova. 